International Journal of Pure and Applied Mathematics

\begin{tabular}{lll}
\hline Volume 94 & No. 1 & $2014,37-44$
\end{tabular}

ISSN: 1311-8080 (printed version); ISSN: 1314-3395 (on-line version)

url: http://www.ijpam.eu

doi: http://dx.doi.org/10.12732/ijpam.v94i1.4

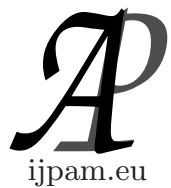

\title{
MATHEMATICAL MODELLING OF MAGNETIC FIELD FROM HETEROGENEOUS MEDIA WITH A HOMOGENEOUS OVERBURDEN
}

\author{
Warin Sripanya \\ Faculty of Science and Technology \\ Nakhon Pathom Rajabhat University \\ Nakhon Pathom, 73000, THAILAND
}

\begin{abstract}
We derive analytical solutions of the steady state magnetic field due to a direct current source on three types of heterogeneous earth structures whose conductivities vary exponentially, linearly and binomially with depth. The Hankel transform is introduced to our problems and analytical results are obtained. Our solutions are achieved by solving a boundary value problem in the wave number domain and then transforming the solution back to the spatial domain. An inverse problem via the use of the Levenberg-Marquardt optimization technique is introduced for finding the conductivity parameters of the ground. The optimal result of our model is close to the true value with percentage errors of our two conductivity parameters less than $2.5 \%$ and $4.3 \%$ after using only 9 iterations.
\end{abstract}

AMS Subject Classification: 86 A25

Key Words: inverse problem, magnetic field, direct current, Hankel transform

\section{Introduction}

The traditional resistivity method maps the electrical properties of the earth by

Received: October 9, 2013

(c) 2014 Academic Publications, Ltd. url: www.acadpubl.eu 
measuring the differences in potential at the earth's surface caused by galvanic current flow between two current electrodes. Many authors have investigated the nature of the resistivity response resulting from a direct current source on a heterogeneous ground whose conductivities vary continuously with depth. Stoyer and Wait [11] firstly considered the conductivity varying exponentially with depth under homogeneous overburden. Banerjee et al. [2], Kim and Lee [5] discussed the problem of a multilayered earth and derived specific case for a two-layered model. In transitional layers, the electrical conductivity is assumed, for simplicity, to be linearly dependent upon depth. This problem was first treated by Mallick and Roy [7], who presented an analysis of the problem of a two-layered earth. Koefoed [6] solved the problem with linear change of the resistivity with depth, a type of change that seems to be more common in nature than the type considered by Mallick and Roy. Banerjee et al. [3] studied the conductivity in a transitional layer which is assumed to be binomially with depth.

In this article, the electrical exploration method based on the measurement of static magnetic fields associated with noninductive current flow between two current electrodes on the earth's surface is introduced. We derive analytical solutions of the steady state magnetic field due to a direct current source on three types of two-layered earth structures with a homogeneous overburden in which the conductivities in a host medium vary exponentially, linearly and binomially with depth. The Hankel transform is introduced to our problems and analytical results are obtained. The inversion process, using the LevenbergMarquardt algorithm, is conducted to estimate the conductivity parameters of the ground.

\section{Model and Basic Equation}

In our geometric model, a point source of direct current $I$ is located at the interface between two half-spaces. The half-space above the interface $(z<0)$ is the region of air with conductivity approximately equal to zero, whereas the half-space below the interface $(z>0)$ is a 2-layered horizontally stratified earth with depth to the layer $h$ (the lowermost layer extending to infinity) measured from the ground surface. Each layer has conductivity as a function of depth, i.e., $\sigma_{k}(z)$ for layer $1 \leq k \leq 2$. The azimuthal component of the magnetic field, denoted by $\tilde{H}$, (Sripanya and Yooyuanyong [10]) can be determined by

$$
\frac{\partial^{2} \tilde{H}}{\partial z^{2}}+\sigma \frac{\partial}{\partial z}\left(\frac{1}{\sigma}\right) \frac{\partial \tilde{H}}{\partial z}-\lambda^{2} \tilde{H}=0 .
$$


Therefore, the magnetic field in each layer can be obtained by taking the inverse Hankel transform (Ali and Kalla [1]) to the solution of equation (1), which satisfies the boundary conditions presented by Sripanya and Yooyuanyong.

\section{Solutions of the Problems}

\subsection{Exponential Profile}

For an exponentially varying conductivity profile, the variation of conductivity is denoted by

$$
\sigma(z)=a e^{b(z-h)},
$$

where $a, b$ and $h$ are constants, which preserve $\sigma(z)>0$. Hence, the magnetic field in an exponentially varying conductive ground can be written as

$$
H(r, z)=\int_{0}^{\infty}\left(A e^{(z-h) \alpha^{-}}+B e^{(z-h) \alpha^{+}}\right) J_{1}(\lambda r) d \lambda,
$$

where

$$
\alpha^{ \pm}=\frac{b \pm \sqrt{b^{2}+4 \lambda^{2}}}{2}
$$

$A$ and $B$ are arbitrary constants, which can be determined by using the boundary conditions.

\subsection{Linear Profile}

For a linearly varying conductivity profile, the variation of conductivity is denoted by

$$
\sigma(z)=a+m(z-h),
$$

where $a, h$ and $m \neq 0$ are constants, which preserve $\sigma(z)>0$. Hence, the magnetic field in a linearly varying conductive ground can be written as

$$
\begin{aligned}
H(r, z)=\int_{0}^{\infty}\left(\psi ( z ) \left(C I_{1}\left(\frac{\lambda}{\varrho} \psi(z)\right)\right.\right. & \\
& \left.\left.+D K_{1}\left(\frac{\lambda}{\varrho} \psi(z)\right)\right)\right) J_{1}(\lambda r) d \lambda
\end{aligned}
$$

where

$$
\varrho=\frac{m}{a}, \quad \psi(z)=1+\varrho(z-h),
$$


$I_{\nu}$ and $K_{\nu}$ are the modified Bessel functions of the first and second kinds of order $\nu$. The unknown coefficients $C$ and $D$ are arbitrary constants, which can be determined by using the boundary conditions.

\subsection{Binomial Profile}

For a binomially varying conductivity profile, the variation of conductivity is denoted by

$$
\sigma(z)=a(1+d(z-h))^{p}
$$

where $a, h, p$ and $d \neq 0$ are constants, which preserve $\sigma(z)>0$. Hence, the magnetic field in a binomially varying conductive ground can be written as

$$
\begin{aligned}
H(r, z)=\int_{0}^{\infty}\left(\tilde { \psi } ^ { \gamma } ( z ) \left(\tilde{C} I_{-\gamma}\left(\frac{\lambda}{\tilde{\varrho}} \tilde{\psi}(z)\right)\right.\right. & \\
& \left.\left.+\tilde{D} K_{-\gamma}\left(\frac{\lambda}{\tilde{\varrho}} \tilde{\psi}(z)\right)\right)\right) J_{1}(\lambda r) d \lambda,
\end{aligned}
$$

where

$$
\tilde{\varrho}=d, \quad \tilde{\psi}(z)=1+\tilde{\varrho}(z-h), \quad \gamma=\frac{1+p}{2},
$$

$\tilde{C}$ and $\tilde{D}$ are arbitrary constants, which can be determined by using the boundary conditions.

\section{2-layered Earth Models}

Let us consider a 2-layered earth model. An overburden has a constant conductivity $a$ with thickness $h$ over a host medium having continuously varying conductivity $\sigma$ as given above.

\subsection{Exponential Profile}

The magnetic fields in an overburden, denoted by $H_{1}$, and in an exponentially varying conductive medium, denoted by $\mathrm{H}_{2}$, can be written as

$$
\begin{gathered}
H_{1}(r, z)=\int_{0}^{\infty} \frac{I}{2 \pi} \frac{\lambda \cosh (\lambda(h-z))-\alpha^{-} \sinh (\lambda(h-z))}{\lambda \cosh (\lambda h)-\alpha^{-} \sinh (\lambda h)} \\
\times J_{1}(\lambda r) d \lambda, \\
H_{2}(r, z)=\int_{0}^{\infty} \frac{I}{2 \pi} \frac{\lambda e^{(z-h) \alpha^{-}}}{\lambda \cosh (\lambda h)-\alpha^{-} \sinh (\lambda h)} J_{1}(\lambda r) d \lambda .
\end{gathered}
$$




\subsection{Linear Profile}

The magnetic fields in an overburden and in a linearly varying conductive medium can be written as

$$
\begin{array}{r}
H_{1}(r, z)=\int_{0}^{\infty} \frac{I}{2 \pi} \frac{\cosh (\lambda(h-z)) K_{1}(\lambda / \varrho)+\sinh (\lambda(h-z)) K_{0}(\lambda / \varrho)}{\cosh (\lambda h) K_{1}(\lambda / \varrho)+\sinh (\lambda h) K_{0}(\lambda / \varrho)} \\
\times J_{1}(\lambda r) d \lambda, \\
H_{2}(r, z)=\int_{0}^{\infty} \frac{I}{2 \pi} \frac{\psi(z) K_{1}((\lambda / \varrho) \psi(z))}{\cosh (\lambda h) K_{1}(\lambda / \varrho)+\sinh (\lambda h) K_{0}(\lambda / \varrho)} \\
\times J_{1}(\lambda r) d \lambda .
\end{array}
$$

\subsection{Binomial Profile}

The magnetic fields in an overburden and in a binomially varying conductive medium can be written as

$$
\begin{gathered}
H_{1}(r, z)=\int_{0}^{\infty} \frac{I}{2 \pi} \frac{\cosh (\lambda(h-z)) K_{\gamma}(\lambda / \varrho)+\sinh (\lambda(h-z)) K_{v}(\lambda / \tilde{\varrho})}{\cosh (\lambda h) K_{\gamma}(\lambda / \varrho)+\sinh (\lambda h) K_{v}(\lambda / \varrho)} \\
\times J_{1}(\lambda r) d \lambda, \\
H_{2}(r, z)=\int_{0}^{\infty} \frac{I}{2 \pi} \frac{\tilde{\psi}^{\gamma}(z) K_{\gamma}((\lambda / \tilde{\varrho}) \tilde{\psi}(z))}{\cosh (\lambda h) K_{\gamma}(\lambda / \tilde{\varrho})+\sinh (\lambda h) K_{v}(\lambda / \tilde{\varrho})} \\
\times J_{1}(\lambda r) d \lambda,
\end{gathered}
$$

where

$$
v=\frac{1-p}{2}
$$

\section{Numerical Experiments and Inversion Process}

In our inverse model example, we simulate the reflection of magnetic radiation data from our forward model of practical interest. The example model is a 2-layered electrically conductive earth. The overburden for our model has a constant conductivity $a$ with thickness $h$ overlying the host medium having 


\begin{tabular}{ccc}
\hline \multicolumn{3}{c}{ Model Parameters } \\
\hline $\begin{array}{c}a\left(\mathrm{~S} \cdot \mathrm{m}^{-1}\right) \\
0.1\end{array}$ & $\frac{b\left(\mathrm{~m}^{-1}\right)}{0.25}$ & $\frac{h(\mathrm{~m})}{5}$ \\
\hline
\end{tabular}

Table 1: Model parameters used in our sample test.

exponentially varying conductivity denoted by $\sigma(z)=a \exp (b(z-h))$ with infinite depth. The values of the model parameters are given in Table ??. Chave's algorithm [4] is used for numerically calculating the inverse Hankel transform of the magnetic field solutions. The special functions are computed by using the Numerical Recipes source codes (Press et al. [9]). Random errors up to $3 \%$ are superimposed on the scaled magnetic fields to simulate the set of real data. The iterative procedure using the Levenberg-Marquardt method (Press et al. [9]) is applied to estimate the model parameters of conductivity variation. The model parameter $a$ is a conductivity of the earth's surface, which can be assumed to be known from the measurement. We start the iterative process to find the values of the conductivity parameters with initial guess values $h=1 \mathrm{~m}$ and $b=1 \mathrm{~m}^{-1}$. The inversion method leads to the optimal values of the parameters $h$ and $b$ with percentage errors less than $2.5 \%$ and $4.3 \%$, respectively, after using only 9 iterations. The graphs of the true and estimated conductivity models are plotted as shown in Figure 1.

\section{Discussions and Conclusions}

Analytical solutions of the steady state magnetic field due to a direct current source are derived for three types of two-layered earth models with a homogeneous overburden in which the conductivities in a host medium vary exponentially, linearly and binomially with depth. The solutions (11) and (12) are applicable to general cases in which the host medium has either constant or exponentially varying conductivity. In transitional ground profiles, the solutions (15) and (16) are generalized in all cases where the medium has constant, linearly or binomially varying conductivity. The model of a simple case for the ground structure is used to investigate the electrical conductivity profile. The iterative procedure using the Levenberg-Marquardt method is applied to estimate the model parameters of conductivity variation. The optimal result of our model converges to the true value with percentage errors of $h$ and $b$ less than $2.5 \%$ and $4.3 \%$, respectively, after using only 9 iterations. The graphs 


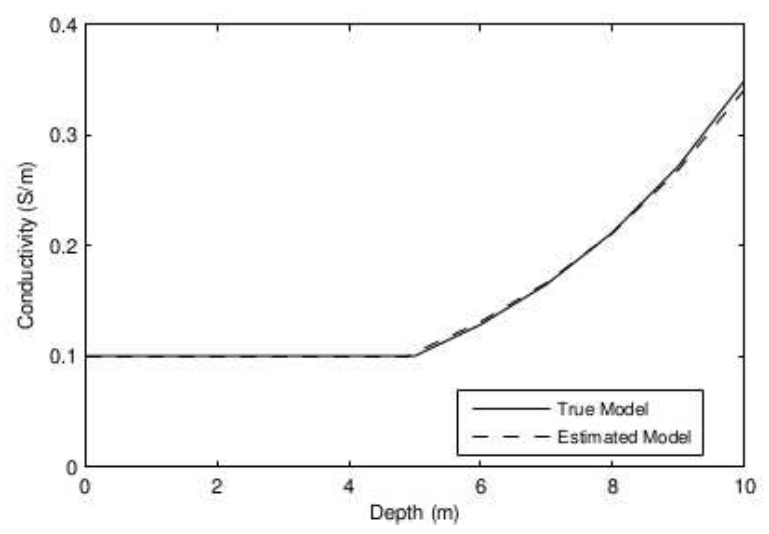

Figure 1: Graphs of conductivity $\sigma$ against depth $z$ for our inverse model example.

of the true and estimated conductivity models are plotted as shown in Figure 1. We clearly see that the graph of the estimated model is close to the true model of conductivity profile. The inversion method leads to very good result and has high speed of convergence. This illustrates the advantage in using the Levenberg-Marquardt method which gives the result much better than using another method of inversion (e.g., Oldenburg [8], Vozoff and Jupp [12]).

\section{References}

[1] I. Ali, S. Kalla, A generalized Hankel transform and its use for solving certain partial differential equations, J. Austral. Math. Soc. Ser. B, 41 (1999), 105-117.

[2] B. Banerjee, B.J. Sengupta, B.P. Pal, Apparent resistivity of a multilayered earth with a layer having exponentiality varying conductivity, Geophys. Prosp., 28 (1980), 435-452.

[3] B. Banerjee, B.J. Sengupta, B.P. Pal, Resistivity sounding on a multilayered earth containing transition layers, Geophys. Prosp., 28 (1980), 750758.

[4] A.D. Chave, Numerical integration of related Hankel transforms by quadrature and continued fraction expansion, Geophysics, 48 (1983), 1671-1686. 
[5] H.-S. Kim, K. Lee, Response of a multilayered earth with layers having exponentially varying resistivities, Geophysics, 61 (1996), 180-191.

[6] O. Koefoed, Resistivity sounding on an earth model containing transition layers with linear change of resistivity with depth, Geophys. Prosp., 27 (1979), 862-868.

[7] K. Mallick, A. Roy, Resistivity sounding on a two-layer earth with transitional boundary, Geophys. Prosp., 16 (1968), 436-446.

[8] D.W. Oldenburg, The interpretation of direct current resistivity measurements, Geophysics, 43 (1978), 610-625.

[9] W.H. Press, S.A. Teukolsky, W.T. Vetterling, B.P. Flannery, Numerical Recipes in Fortran 7\%: The Art of Scientific Computing, Fortran Numerical Recipes Vol. 1, 2nd ed., Cambridge Univ. Press (1992).

[10] W. Sripanya and S. Yooyuanyong, Mathematical inverse problem of magnetic field from heterogeneous earth structures, Far East J. Math. Sci., 70 (2012), 187-199.

[11] C.H. Stoyer, J.R. Wait, Resistivity probing of an "exponential" earth with a homogeneous overburden, Geoexpl., 15 (1977), 11-18.

[12] K. Vozoff, D.L.B. Jupp, Joint inversion of geophysical data, Geophys. J. R. astr. Soc., 42 (1975), 977-991. 